\title{
A Brief Look at the Chirality-Flow Formalism for Standard Model Amplitudes
}

\author{
Joakim Alnefjord, ${ }^{a}$ Andrew Lifson, ${ }^{a, *}$ Christian Reuschle ${ }^{a}$ and Malin Sjodahl ${ }^{a}$ \\ ${ }^{a}$ Department of Astronomy and Theoretical Physics, Lund University, \\ Sölvegatan 14A, 22362 Lund, Sweden \\ E-mail: joakim.alnefjord@gmail.com, andrew.lifson@thep.lu.se, \\ christian.reuschle@thep.lu.se, malin.sjodahl@thep.lu.se
}

Inspired by the flow description of su(N) colour calculations, we recently showed how to simplify the spinor-helicity formalism (at the algebra level two copies of complexified su(2)) by treating each Weyl spinor as part of a flow line with definite chirality and momentum. This formalism, dubbed the chirality-flow formalism, eliminates all non-trivial algebra from tree-level spinorhelicity calculations, thus allowing the shortest possible route from Feynman diagrams to complex numbers (spinor inner products). In this presentation, we briefly introduce the main features of this method and show some examples.

The Ninth Annual Conference on Large Hadron Collider Physics - LHCP2021

7-12 June 2021

Online

${ }^{*}$ Speaker 


\section{Introduction and the massless spinor-helicity formalism}

The spinor-helicity formalism is often the most convenient framework in which to perform scattering amplitude calculations [1-25]. At its core, it describes particles as (combinations of) two-component Weyl spinors which transform separately under Lorentz transformations. At the level of the Lorentz algebra $s o(3,1)_{\mathbb{C}} \cong s u(2)_{\mathbb{C}, L} \oplus s u(2)_{\mathbb{C}, R}$, the Weyl spinors transform under either the left-chiral $s u(2)_{\mathbb{C}, L}$ or the right-chiral $s u(2)_{\mathbb{C}, R}$. For example, we can make this decomposition manifest by considering Dirac spinors in the chiral basis, written schematically (for some $p_{1}, p_{2}$ ) as

$$
u(p) \sim v(p) \sim\left(\begin{array}{l}
\left.\mid p_{1}\right] \\
\left|p_{2}\right\rangle
\end{array}\right) \quad \bar{u}(p) \sim \bar{v}(p) \sim\left(\left[p_{1} \mid,\left\langle p_{2}\right|\right) \quad \gamma^{5}=\left(\begin{array}{cc}
-1 & 0 \\
0 & 1
\end{array}\right),\right.
$$

where the square brackets are Weyl spinors transforming under $s u(2)_{\mathbb{C}, L}$, the angled brackets are Weyl spinors transforming under $s u(2)_{\mathbb{C}, R}$, and the eigenvalue of $\gamma^{5}$ gives the chirality.

Since helicity is the spin-quantum number of any massless particle [26-28], it is natural to calculate massless scattering amplitudes using states of definite helicity. For massless Weyl spinors, such states are also eigenstates of the chirality operator $\gamma^{5}$, meaning they transform under only one $s u(2)$

$$
\begin{array}{ll}
u^{+}(p)=v^{-}(p)=\left(\begin{array}{c}
0 \\
|p\rangle
\end{array}\right) & u^{-}(p)=v^{+}(p)=\left(\begin{array}{c}
\mid p] \\
0
\end{array}\right), \\
\bar{u}^{+}(p)=\bar{v}^{-}(p)=([p \mid, 0) & \bar{u}^{-}(p)=\bar{v}^{+}(p)=(0,\langle p|) .
\end{array}
$$

Conversely, using $\tau^{\mu}=(1, \vec{\sigma}) / \sqrt{2}$ and $\bar{\tau}^{\mu}=(1,-\vec{\sigma}) / \sqrt{2}$, vectors can be seen as containing both chiralities, with massless momenta given by

$$
\left.\sqrt{2} p^{\mu} \tau_{\mu} \equiv \not p=\mid p\right]\left\langle p\left|, \quad \sqrt{2} p^{\mu} \bar{\tau}_{\mu} \equiv \bar{p}=\right| p\right\rangle[p \mid,
$$

and outgoing gauge bosons given by $[9,14]$

$$
\epsilon_{+}^{\mu}(p, r)=\frac{\left\langle r\left|\bar{\tau}^{\mu}\right| p\right]}{\langle r p\rangle}, \quad \quad \epsilon_{-}^{\mu}(p, r)=\frac{\left[r\left|\tau^{\mu}\right| p\right\rangle}{[p r]} .
$$

Here, the gauge boson has momentum $p$, while $r$ is an arbitrary reference momentum which corresponds to a particular gauge choice.

After using algebraic identities such as (see for example [29, 30])

$$
\underbrace{\left\langle i\left|\bar{\tau}^{\mu}\right| j\right]\left[k\left|\tau_{\mu}\right| l\right\rangle=\langle i l\rangle[k j]}_{\text {Fierz identity }} \quad \text { and } \quad \underbrace{\left\langle i\left|\bar{\tau}^{\mu}\right| j\right]=\left[j\left|\tau^{\mu}\right| i\right\rangle}_{\text {charge conjugation }},
$$

a scattering amplitude is written in terms of Lorentz-invariant spinor inner products

$$
\langle i j\rangle=-\langle j i\rangle \equiv\langle i \| j\rangle \quad \text { and } \quad[i j]=-[j i] \equiv[i \| j], \quad\langle i j\rangle \sim[i j] \sim \sqrt{2 p_{i} \cdot p_{j}}
$$

which are simple, well known complex numbers. 


\section{Chirality flow}

In the last section we saw that with a few algebraic identities one can move from Feynman rules to complex numbers. In this section we describe a set of flow rules which eliminates the need for explicitly using these identities, which are instead built into the flow rules [31-33].

We begin with an ansatz for the spinor inner products. Since the left- and right-chiral states transform separately under Lorentz transformations, we require two distinct line types. Inspired by square brackets having dotted indices and angled brackets having undotted indices, we use dotted (more accurately dashed) lines to refer to square inner products, and solid lines for angled inner products

$$
\left\langle\left. i\right|^{\alpha} \mid j\right\rangle_{\alpha} \equiv\langle i j\rangle=-\langle j i\rangle=i \longrightarrow \quad j \quad, \quad\left[\left.i\right|_{\dot{\beta}} \mid j\right]^{\dot{\beta}} \equiv[i j]=-[j i]=i \ldots \ldots \ldots j,
$$

where the arrow direction matters since the inner products are antisymmetric. Cutting the flow lines in two gives the flow definitions of the spinors

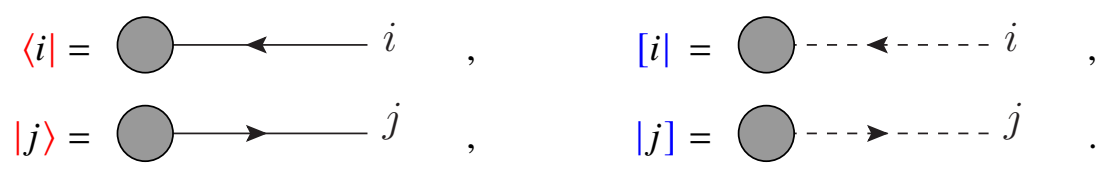

In [31], we proved that we can always use the Fierz identity on the Pauli matrices, thus replacing a vector with a chirality-flow double line, i.e. a solid and dotted line with arrows opposing

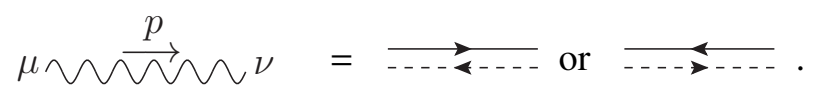

Finally, we define the momentum dot for slashed momenta (with $p=\sum_{i} p_{i}, p_{i}^{2}=0$ )

$$
\sqrt{2} p^{\mu} \bar{\tau}_{\mu}=\sum_{i}|i\rangle\left[i\left|=\longrightarrow \stackrel{\Sigma_{i} p_{i}}{\longrightarrow} \mathbf{\longrightarrow}_{--}, \quad \sqrt{2} p^{\mu} \tau_{\mu}=\sum_{i}\right| i\right]\langle i|=\ldots \stackrel{\Sigma_{i} p_{i}}{\longrightarrow} .
$$

If the particles are massive, we describe them as combinations of massless spinors allowing to recycle the above results. For instance, a massive momentum $p$ with $p^{2}=m^{2} \neq 0$ is decomposed as a sum of massless momenta $p^{b}$ and $q$

$$
p^{\mu}=p^{b, \mu}+\alpha q^{\mu}, \quad\left(p^{b}\right)^{2}=q^{2}=0, \quad p^{2}=m^{2}, \quad \alpha=\frac{m^{2}}{2 p^{b} \cdot q}=\frac{m^{2}}{2 p \cdot q},
$$

while, for example, an incoming spinor with spin along the axis $s^{\mu}=\left(p^{\mu}-2 \alpha q^{\mu}\right) / m$ is given by

$$
u^{+}(p)=\left(\begin{array}{r}
\left.-e^{-i \varphi} \sqrt{\alpha} \mid q\right] \\
\left|p^{b}\right\rangle
\end{array}\right)=\left(\begin{array}{r}
-e^{-i \varphi} \sqrt{\alpha} \bigcirc \ldots-q^{b} \\
\bigcirc
\end{array}\right), \quad e^{-i \varphi} \sqrt{\alpha}=\frac{m}{\left[q p^{b}\right]} .
$$

A full list of massive spinors and polarisation vectors, together with the Standard Model flow rules is given in [33]. 


\section{Standard Model examples}

To calculate a Feynman diagram in massless QED, we simply draw the chirality-flow lines without the arrows, then connect them as given by the flow rules. Next, we choose a single arrow direction and follow it through the diagram, remembering the requirement of opposing arrows for a double line, eq. (2.3). This process leads to an algebra-free journey from a Feynman diagram to inner products for even very complicated diagrams such as (Feynman in black, flow lines in colour, all momenta outgoing)

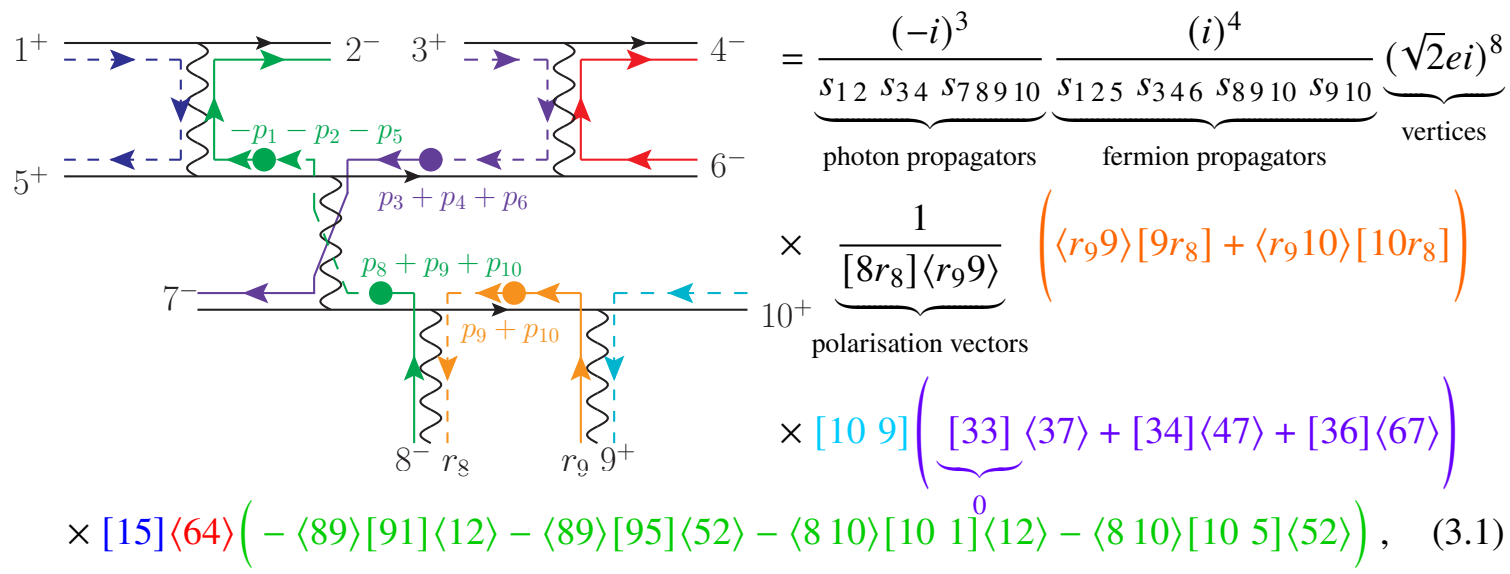

where the flow line and inner product colours coincide and the black prefactors are trivially found.

When using massive fermions, we have more components in our flow rules, both from the external spinors (e.g. eq. (2.6)) and from the mass term in the fermion propagator. We then build the flow diagram from the flow rules as in eq. (3.1), but have to take care of minus signs [33]. For example, we find (ignoring trivial factors)

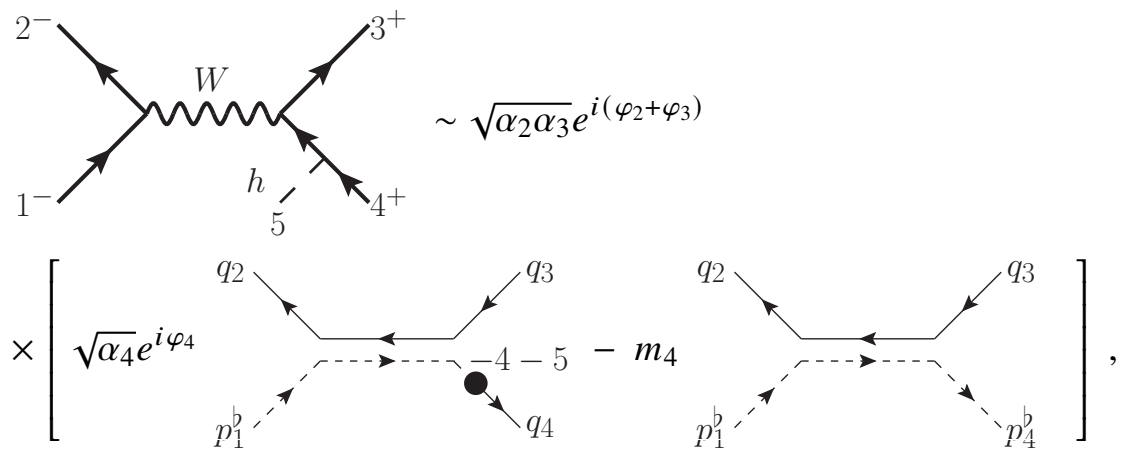

where the flow lines are the inner products we seek, the weak interaction simplifies by removing right-chiral couplings, and the Higgs has no flow since it is a Lorentz scalar.

\section{Conclusions and outlook}

In this presentation we reviewed the basics of the novel chirality-flow method, which allows to go from Feynman diagrams to complex numbers without intermediate algebraic manipulations. We gave examples of massless and massive tree-level Feynman diagrams to illustrate the efficiency and transparency of our method, which can be used to calculate any tree-level Standard Model process. In future, we aim to extend this method to loops and recursive calculations. 


\section{References}

[1] P. De Causmaecker, R. Gastmans, W. Troost and T.T. Wu, Multiple Bremsstrahlung in Gauge Theories at High-Energies. 1. General Formalism for Quantum Electrodynamics, Nucl. Phys. B206 (1982) 53.

[2] F.A. Berends, R. Kleiss, P. De Causmaecker, R. Gastmans and T.T. Wu, Single Bremsstrahlung Processes in Gauge Theories, Phys. Lett. 103B (1981) 124.

[3] F.A. Berends, R. Kleiss, P. De Causmaecker, R. Gastmans, W. Troost and T.T. Wu, Multiple Bremsstrahlung in Gauge Theories at High-Energies. 2. Single Bremsstrahlung, Nucl. Phys. B206 (1982) 61.

[4] P. De Causmaecker, R. Gastmans, W. Troost and T.T. Wu, Helicity Amplitudes for Massless QED, Phys. Lett. 105B (1981) 215.

[5] CALKUL collaboration, Multiple Bremsstrahlung in Gauge Theories at High-energies. 3. Finite Mass Effects in Collinear Photon Bremsstrahlung, Nucl. Phys. B239 (1984) 382.

[6] R. Kleiss, The Cross-section for $e^{+} e^{-} \rightarrow e^{+} e^{-} e^{+} e^{-}$, Nucl. Phys. B241 (1984) 61.

[7] F.A. Berends, P.H. Daverveldt and R. Kleiss, Complete Lowest Order Calculations for Four Lepton Final States in electron-Positron Collisions, Nucl. Phys. B253 (1985) 441.

[8] J.F. Gunion and Z. Kunszt, Four jet processes: gluon-gluon scattering to nonidentical quark - anti-quark pairs, Phys. Lett. 159B (1985) 167.

[9] J.F. Gunion and Z. Kunszt, Improved Analytic Techniques for Tree Graph Calculations and the G g q anti-q Lepton anti-Lepton Subprocess, Phys. Lett. 161B (1985) 333.

[10] R. Kleiss and W.J. Stirling, Spinor Techniques for Calculating $p$ anti-p $\rightarrow W^{ \pm} / Z^{0}+$ Jets, Nucl. Phys. B262 (1985) 235.

[11] K. Hagiwara and D. Zeppenfeld, Helicity Amplitudes for Heavy Lepton Production in e+eAnnihilation, Nucl. Phys. B274 (1986) 1.

[12] R. Kleiss, Hard Bremsstrahlung Amplitudes for $e^{+} e^{-}$Collisions With Polarized Beams at LEP / SLC Energies, Z. Phys. C33 (1987) 433.

[13] R. Kleiss and W.J. Stirling, Cross-sections for the Production of an Arbitrary Number of Photons in Electron - Positron Annihilation, Phys. Lett. B179 (1986) 159.

[14] Z. Xu, D.-H. Zhang and L. Chang, Helicity Amplitudes for Multiple Bremsstrahlung in Massless Nonabelian Gauge Theories, Nucl. Phys. B291 (1987) 392.

[15] CALKUL collaboration, New techniques and results in gauge theory calculations, in Electroweak effects at high-energies. Proceedings, 1st Europhysics study conference, Erice, Italy, February 1-12, 1983, pp. 599-609, 1987. 
[16] C. Schwinn and S. Weinzierl, Scalar diagrammatic rules for Born amplitudes in QCD, JHEP 05 (2005) 006 [hep-th/0503015].

[17] G.R. Farrar and F. Neri, How to Calculate $35640 O\left(\alpha^{5}\right)$ Feynman Diagrams in Less Than an Hour, Phys. Lett. 130B (1983) 109.

[18] F.A. Berends and W. Giele, The Six Gluon Process as an Example of Weyl-Van Der Waerden Spinor Calculus, Nucl. Phys. B294 (1987) 700.

[19] F.A. Berends and W.T. Giele, Recursive Calculations for Processes with $n$ Gluons, Nucl. Phys. B306 (1988) 759.

[20] F.A. Berends, W.T. Giele and H. Kuijf, Exact Expressions for Processes Involving a Vector Boson and Up to Five Partons, Nucl. Phys. B321 (1989) 39.

[21] F.A. Berends and W.T. Giele, Multiple Soft Gluon Radiation in Parton Processes, Nucl. Phys. B313 (1989) 595.

[22] F.A. Berends, W.T. Giele and H. Kuijf, Exact and Approximate Expressions for Multi Gluon Scattering, Nucl. Phys. B333 (1990) 120.

[23] S. Dittmaier, Full O(alpha) radiative corrections to high-energy Compton scattering, Nucl. Phys. B423 (1994) 384 [hep-ph/9311363].

[24] S. Dittmaier, Weyl-van der Waerden formalism for helicity amplitudes of massive particles, Phys. Rev. D59 (1998) 016007 [hep-ph/9805445].

[25] S. Weinzierl, Automated computation of spin- and colour-correlated Born matrix elements, Eur. Phys. J. C45 (2006) 745 [hep-ph/0510157].

[26] E.P. Wigner, On Unitary Representations of the Inhomogeneous Lorentz Group, Annals Math. 40 (1939) 149.

[27] V. Bargmann and E.P. Wigner, Group Theoretical Discussion of Relativistic Wave Equations, Proc. Nat. Acad. Sci. 34 (1948) 211.

[28] S. Weinberg, The Quantum theory of fields. Vol. 1: Foundations, Cambridge University Press $(6,2005)$.

[29] L.J. Dixon, Calculating scattering amplitudes efficiently, in $Q C D$ and beyond. Proceedings, Theoretical Advanced Study Institute in Elementary Particle Physics, TASI-95, Boulder, USA, June 4-30, 1995, pp. 539-584, 1996 [hep-ph/9601359].

[30] H. Elvang and Y.-t. Huang, Scattering Amplitudes, 1308 . 1697.

[31] A. Lifson, C. Reuschle and M. Sjodahl, The chirality-flow formalism, Eur. Phys. J. C 80 (2020) 1006 [2003.05877].

[32] A. Lifson, C. Reuschle and M. Sjödahl, Introducing the Chirality-flow Formalism, Acta Phys. Polon. B 51 (2020) 1547. 
[33] J. Alnefjord, A. Lifson, C. Reuschle and M. Sjodahl, The chirality-flow formalism for the standard model, Eur. Phys. J. C 81 (2021) 371 [2011. 10075]. 